EGU2020-7468

https://doi.org/10.5194/egusphere-egu2020-7468

EGU General Assembly 2020

(c) Author(s) 2020. This work is distributed under

the Creative Commons Attribution 4.0 License.

\title{
Effects of an abrupt depth change on weakly nonlinear surface gravity waves: deterministic and stochastic analysis
}

\author{
Yan $\mathbf{L i}^{1,3}$, Samuel Draycott ${ }^{2}$, Yaokun Zheng ${ }^{4}$, Thomas A.A. Adcock ${ }^{1}$, Zhiliang Lin ${ }^{4}$, and Ton S. van den \\ Bremer $^{1}$ \\ ${ }^{1}$ Department of Engineering Science, University of Oxford, Parks Road, Oxford OX1 3PJ, UK; \\ ${ }^{2}$ Department of Mechanical, Aerospace \& Civil Engineering, University of Manchester, Manchester, UK; \\ ${ }^{3}$ Norwegian University of Science and Technology, Department of energy and process engineering, 7491 Trondheim, \\ Norway \\ ${ }^{4}$ State Key Laboratory of Ocean Engineering, School of Naval Architecture, Ocean and Civil Engineering, Shanghai Jiao Tong \\ University, 200240, China
}

This work focuses on two different aspects of the effect of an abrupt depth transition on weakly nonlinear surface gravity waves: deterministic and stochastic. It is known that the kurtosis of waves can reach a maximum near the top of such abrupt depth transitions. The analysis is based on three different approaches: (1) a novel theoretical framework that allows for narrow-banded surface waves experiencing a step-type seabed, correct to the second order in wave steepness; (2) experimental observations; and (3) a numerical model based on a fully nonlinear potential flow solver. To reveal the fundamental physics, the evolution of a wave envelope that experiences an abrupt depth transition is examined in detail; (a) we show the release of free waves at second order in wave steepness both for the super-harmonic and sub-harmonic or 'mean' terms; (b) a local wave height peak that occurs near the top of a depth transition - whose exact position depends on several nondimensional parameters - is revealed; (c) furthermore, we examine which parameters affect this peak. The novel physics has implications for wave statistics for long-crested irregular waves experiencing an abrupt depth transition. We show the connection of the secondorder physics at work in the deterministic and stochastic cases: the peak of wave kurtosis and skewness occurs in the neighborhood of the deterministic wave peak in (b) and for the same parameters set composed of a seabed topography, water depths, primary wave frequency and steepness, and bandwidth. 